\title{
Dilemas e frustrações do estudante de medicina: estudo transversal
}

\author{
Dilemmas and frustrations of medical students: a cross-sectional study \\ Daniel Haber Feijó', Carolina Ribeiro Mainardi', André Lopes Valente', Vitor Nagai Yamaki', \\ Renan Kleber Costa Teixeira ${ }^{1}$, José Antônio Cordero da Silva ${ }^{1}$
}

Resumo Introdução: O período da graduação no curso de medicina é fundamental para a construção científica, psicológica e ética do futuro médico. O aluno passa por muitas mudanças, que podem ter consequências catastróficas se não houver adequada condução do aluno por parte da docência. Assim buscou-se identificar os dilemas e frustrações do estudante de medicina. Método: Foi realizado um estudo transversal nos estudantes de medicina do $1^{\circ}$ o $4^{\circ}$ ano da Universidade do Estado do Pará. Foram entrevistados 100 alunos divididos igualmente por cada ano do curso, por meio de questionário autoaplicável para avaliar os dilemas e frustações do estudante de medicina. Resultados: 95\% dos alunos foram classificados com algum grau de frustração com o curso de medicina. Dentre as queixas mais comuns encontramos o desânimo com o curso ( $93 \%$ ), decepção (76\%), falta de orientação pelos professores (97\%) e sobrecarga (97\%). Foi encontrado um aumento do consumo de álcool e tabaco conforme os estudantes avançam no curso. Discussão: A frustração com o curso de medicina foi evidente nesta pesquisa, independente de fatores como sexo, idade, uso de drogas ou doenças, iniciando em períodos precoces do curso. O desânimo com a faculdade tem sido associado ao desenvolvimento de cinismo, pessimismo e burnout profissional. Conclusão: Os estudantes de medicina mostraram-se frustrados com o curso, sendo os principais dilemas a falta de orientação por parte dos professores e a sobrecarga de atividades acadêmicas.

Descritores: estudantes de medicina; ansiedade; estresse; empatia.

Summary Introduction: The graduation period in the medical school is fundamental to the future scientific, psychological and ethical formation. The student goes through many changes that can have catastrophic consequences if no proper conduction of the student is performed by the professors Therefore, we sought to identify the dilemmas and frustrations of the medical students. Method: It was performed a cross-sectional study. We interviewed 100 students enrolled in the course of Medicine from the Pará State University, at the Belem Unit, distributed equally between the first 4 years of graduation, using a self-administered questionnaire to assess the dillemas and frustration of the medical students. Results: $95 \%$ had some level of frustration with the medical school. The most common complaints were discouragement with course, disappointment (76\%), lack of orientation by professors (97\%) and overload (97\%). In relation to drug consumption, we found an increasing consumption of alcohol and tobacco throughout the course. Discussion: The frustration with the medical course was evident in this research, independently of factors such as gender, age, drug use or disease, starting early in the course periods. Discouragement with the college has been associated with the development of cynicism, pessimism and professional burnout. Conclusion: The students demonstrated frustration with the course mainly because of the lack of orientation by the professors and overwhelming academic activities.

Keywords: students, medical; anxiety; stress; empathy.

'Universidade do Estado do Pará - UEPA, Belém, Pará, Brasil

Fonte de financiamento: Nenhuma.

Conflito de interesses: Os autores declaram não haver conflitos de interesse.

Recebido: Junho 28, 2018

Aceito: Julho 12, 2018

Trabalho realizado na Universidade do Estado do Pará, em Belém, Pará, Brasil.

Copyright Feijó et al. Este é um artigo publicado em acesso aberto (Open Access) sob a licença Creative Commons Attribution, que permite uso, distribuição e reprodução em qualquer meio, sem restrições desde que o trabalho original seja corretamente citado. 


\section{Introdução}

O período da graduação no curso de medicina é fundamental para a construção científica, psicológica e ética do futuro médico ${ }^{1}$. O acadêmico de medicina vive uma completa mudança de realidade comparada à formação do Ensino Médio. Tais mudanças podem ter consequências catastróficas se não houver adequada condução do aluno e acompanhamento por parte da docência².

Nesse sentido, o estudante desenvolve diferentes modos de enfrentamento às situações adversas. Aqueles com personalidade estruturada e bom respaldo familiar tendem a se aprimorar às dificuldades. Já aqueles com menor capacidade de resiliência, encontram muitos obstáculos e até mesmo desenvolvem desordens psicológicas ${ }^{3,4}$.

Os principais dilemas dos alunos de medicina incluem a adequação da vida ao ambiente da faculdade, os conflitos éticos vivenciados junto aos pacientes e professores, assim como a exposição à morte e sofrimento do ser humano e até mesmo os eventos pessoais e familiares como ocorrência de doenças 5 . Infelizmente, esses aspectos do processo de formação geram consequências negativas à saúde pessoal do estudante levando a um quadro de frustração e desânimo com o curso ${ }^{5,6}$.

Estudos de revisão sistemática apontam que $28 \%$ dos estudantes possuem sintomas depressivos ${ }^{7} \mathrm{e}$ $93 \%$ cursam com ansiedade ${ }^{8}$, bem como índices de ideação suicida de até $14 \%{ }^{9}$. Além disso, alterações na qualidade do sono estão presentes em até $60 \%$ dos estudantes ${ }^{10}$, assim como abuso de drogas ou álcool está em torno de $20 \% \%^{8,11}$.

Tendo em vista a importância de estudar os conflitos vivenciados pelo estudante a fim de tentar esclarecer quais os principais motivos que levam à frustração com o curso, esta pesquisa objetiva identificar os principais dilemas e frustrações dos estudantes de medicina de uma universidade pública.

\section{Método}

A presente pesquisa foi iniciada após aprovação pelo Comitê de Ética em Pesquisa da Universidade do Estado do Pará (Parecer 247.571/2013). Este trabalho caracteriza-se como transversal e observacional, a casuística foi determinada por cálculo estatístico que determinou o mínimo de 23 alunos por ano, sendo então definido o número de 25 alunos, do $1^{\circ}$ ao $4^{\circ}$ ano do curso de medicina, selecionados aleatoriamente, para os quais foram aplicadas entrevistas baseadas em um questionário padronizado. Antes da aplicação desses questionários, foi solicitada a assinatura do termo de consentimento livre e esclarecido.

Os critérios de inclusão foram discentes regularmente matriculados, de ambos os sexos e com idade acima de 18 anos. Os critérios de não inclusão foram alunos que já cursaram outros cursos, que repetiram algum ano, transferidos de outras faculdades ou aqueles que não quiseram participar da pesquisa. Não houve critério de exclusão.

As entrevistas foram realizadas no período de Outubro a Novembro de 2014, fora do período avaliativo curricular e em local reservado. O protocolo de pesquisa foi previamente validado com um estudo piloto com 15 alunos do internato. O questionário consistia em duas partes: 1) Dados pessoais (sexo, idade, ano do curso, uso de drogas, sintomas relacionados frustação, necessidade de avaliação e/ou tratamento desses sintomas) e 2) Avaliação do grau de frustações: Foram utilizados 12 questionamentos acerca da percepção do discente sobre: o curso, os professores e outros dilemas do cotidiano do curso. As respostas eram baseadas em "sim" ou "não". Com base nessas respostas os alunos foram classificados em sem frustação (0 a 2 pontos), leve frustação (3-7 pontos) e grande frustação (7-12 pontos).

Foi utilizado o software BioEstat ${ }^{\circledR} 5.4$ para a realização da análise estatística. O teste $T$ de student foi utilizado para comparar a média das idades entre o sexo e o teste Contingência em C para comparar o grau de frustração dos alunos com os dados pessoais dos discentes. Foi adotado um p-valor menor que $5 \%$ para rejeitar a hipótese de nulidade.

\section{Resultados}

De um total de 412 discentes, 100 alunos fizeram parte da pesquisa, do 1 ao 4 ano da faculdade de medicina, sendo 25 alunos de cada ano. A média de idade dos alunos foi de 21 anos, sendo que $56 \%$ eram do sexo feminino. Não houve diferença entre as medias de idades entre homens e mulheres $(\mathrm{p}=0,58)$.

Dentre os analisados, 48 alunos utilizam ou utilizaram drogas; a principal droga utilizada foi o álcool por $43(89 \%)$ discentes, seguida do tabaco $(12-25 \%)$ e maconha $(3-6,25 \%) .69 \%$ dos estudados tinham apenas 
Tabela 1. Resposta dos alunos da Universidade do Estado do Pará ao questionário por quesito relacionado aos possíveis dilemas e frustrações

\begin{tabular}{|c|c|c|c|c|c|}
\hline \multirow{2}{*}{ Quesitos } & \multicolumn{5}{|c|}{ Resposta positiva } \\
\hline & $1^{\circ}$ ano & $2^{\circ}$ ano & $3^{\circ}$ ano & $4^{\circ}$ ano & Total \\
\hline Decepção com o curso & 19 & 18 & 19 & 20 & 76 \\
\hline Desânimo com o curso & 22 & 24 & 23 & 24 & 93 \\
\hline Sobrecarregado & 25 & 24 & 24 & 24 & 97 \\
\hline Falta de orientação pelos professores & 25 & 24 & 25 & 23 & 97 \\
\hline Cobrança excessiva & 20 & 15 & 18 & 18 & 71 \\
\hline Cobrança familiar & 9 & 7 & 9 & 9 & 34 \\
\hline Cobrança dos pacientes* & 0 & 2 & 12 & 12 & 34 \\
\hline Insegurança ao atender & 21 & 22 & 24 & 22 & 89 \\
\hline Distanciamento do curso & 7 & 8 & 9 & 7 & 30 \\
\hline Afetou a vida social & 22 & 23 & 19 & 20 & 84 \\
\hline Dificuldade de relacionamentos* & 22 & 12 & 12 & 10 & 56 \\
\hline Outras alterações & 10 & 8 & 6 & 6 & 30 \\
\hline
\end{tabular}

${ }^{*} \mathrm{p}<0,05$ (T de student).

um vicio, $25 \%$ dois vícios e $4 \%$ três vícios. Houve uma diferença significativa na prevalência de alunos que utiliza cigarro $(\mathrm{p}<0,01)$ ou álcool $(\mathrm{p}=0,04)$ entre o 1 e 2 ano do curso versus o 3 e 4 ano.

Em relação à presença de sintomas relacionados à frustação, 14 pessoas não apresentaram nenhum dentre as listadas. Os sintomas mais comuns foram fadiga ( $46-53 \%)$ e ansiedade $(41-48 \%)$, seguidos de alterações do sono (35-40\%) e gastrointestinais $(35-40 \%)$ e outros $(23-27 \%)$.

Do total de pessoas com algum sintoma, 37 (43\%) buscaram atendimento profissional para tratarem suas doenças. Desse universo a maioria aderiu aos tratamentos propostos pelos profissionais de saúde (31-84\%).

Em relação a frustação com o curso, 5 pessoas não apresentaram frustação, 59 apresentaram uma frustação leve e 36 uma grande frustação com o curso. Sendo que essa frustação não foi influenciada pelo sexo $(p=0,39)$, idade $(p=0,43)$, semestre $(p=0,15)$, uso de drogas $(p=0,17)$ ou presença de patologias $(p=0,22)$. A Tabela 1 descreve cada quesito da escala de frustação. Não houve diferença entre os quesitos avaliados de acordo com as variáveis analisadas ( $p>0,05)$, com exceção da cobrança dos pacientes, que foi maior no terceiro e quarto ano em relação ao primeiro e segundo ano $(p=0,03)$.

\section{Discussão}

A frustração com o curso de medicina foi evidente nesta pesquisa independente de fatores como sexo, idade, uso de drogas ou doenças, iniciando em períodos precoces do curso. O desânimo com a faculdade tem sido associado ao desenvolvimento de cinismo, pessimismo e burnout profissional precocemente, que constituem sérias consequências para o estudante ${ }^{8,12}$, podendo necessitar de assistência médica ${ }^{13,14}$.

O grau de frustração não variou quanto ao gênero, apesar da maioria dos trabalhos indicarem que o gênero feminino é mais diagnosticado com sintomas de depressão e ansiedade ${ }^{7,8,12}$. Semelhante ao encontrado nos estudos de coorte de Tyssen et al. $(2001)^{9}$ e Niemi et al. $(2006)^{15}$, acreditamos que a própria formação e sua progressão durante a longa jornada são mais relevantes que o fator gênero, atingindo em igual intensidade ambos os sexos.

Não houve diferença significativa entre o grau de frustação e o período no curso, contudo sabe-se que há diferentes fatos que podem gerar os dilemas e frustações nos acadêmicos de medicina nesses distintos períodos.

Nos primeiros anos da faculdade, os estudantes de medicina ingressam no chamado ciclo básico, onde há pouco contato com pacientes e há uma vivencia predominante em laboratórios. Dessa forma, o estudo 
das ciências básicas representa uma quebra de paradigmas com a realidade evidenciada e o imaginário dos discentes que ingressam no curso de medicina com o imaginário exibido em filmes ou livros ${ }^{16,17}$.

Além disso, os estudantes aprovados para o curso de medicina, em vários casos, devido o desgaste e dedicação pessoal para alcançar a vaga na universidade iniciam seus estudos esgotados, em que satisfação de passar em um dos cursos mais concorridos entrelaça-se com vários mitos e projeções pessoais e familiares do que vem a ser a formação e a profissão medica.

Em relação à realidade do terceiro e quarto ano, quando se inicia o ciclo clínico começa o grande contato com os pacientes e o inicio das cobranças pelos pacientes e professores com relação aos conhecimentos já adquiridos, além de um aumento da cobrança familiar e pessoal. Nesse período também há maior acúmulo de disciplinas cursadas e o aluno começa a lidar com conflitos éticos como pacientes em estado terminal, dor e sofrimento humano e situações relacionadas ao mau tratamento dos pacientes por parte dos professores e médicos ${ }^{18,19}$. Tudo isso pode corroer a autoestima do aluno de medicina.

Apesar do álcool ter sido a droga mais consumida pelos alunos, a sua prevalência foi menor em comparação a outros estudos com estudantes de medicina, realizados em Minas Gerais ${ }^{20}$ e Alagoas ${ }^{21}$, onde houve uma prevalência entre 64 e 90\% da amostra. Tanto o abuso de álcool quanto tabaco tendem a serem práticas perpetuadas para a profissão médica e em até $12 \%$ evoluem para a dependência ${ }^{22,23}$. Ainda que nosso estudo não tenha evidenciado relação entre o consumo drogas e os conflitos vivenciados no curso médico, percebemos que houve um aumento na prevalência nos semestres mais avançados. Em estudo de coorte conduzido em 2001, a média de consumo de álcool aumentou durante os 4 anos analisados ${ }^{22}$, sendo os níveis de estresse, provas e tensão comumente relatados como causas de consumo ${ }^{24}$. Contudo, não foi avaliada uma possível relação entre a quantidade consumida de álcool e os quesitos de frustração, o que merece melhor investigação em futuros estudos.

A insatisfação e decepção dos alunos de medicina constatada em nossa pesquisa é algo relatado na literatura. Poucos estudos nacionais se dedicaram a entender as causas da decepção e dos dilemas enfrentados pelos estudantes de medicina, a maioria destes em abordagens qualitativas ${ }^{16,25}$.

Em estudo realizado numa faculdade pública do Paquistão com realidade próxima ao nosso sistema público, $58 \%$ dos alunos estavam insatisfeitos com a qualidade de ensino ${ }^{26}$. Outros estudos relatam que o abuso psicológico na formação do médico, quando presente, é um dos principais fatores de estresse e desânimo $0^{5,27}$.

Sabe-se que as consequências para os estudantes quando insatisfeitos e frustrados, segundo estudos, é a maior susceptibilidade a problemas acadêmicos e pessoais como sintomas depressivos, desonestidade acadêmica, menor cuidado com a saúde pessoal, alterações nos relacionamentos ${ }^{5,28}$. Aqueles que enfrentam maiores dificuldades com o curso e pior performance acadêmica, tendem a abandoná-lo, segundo revisão sistemática que avaliou as causas de desistências no curso de medicina ${ }^{29}$.

Outra importante consequência em longo prazo é quanto à influência desses eventos durante o período de graduação na empatia do futuro profissional. Maioria dos estudos concorda que quando os alunos são expostos a mais sofrimento emocional e tem a qualidade de vida afetada pelo curso, há piora do cuidado ao paciente e diminuição da empatia ${ }^{30}$.

O dado mais preocupante do estudo está na queixa de falta de orientação por parte dos professores. Apesar de existir um serviço de apoio psicopedagógico ao estudante de medicina, previsto na lei de diretrizes e bases da educação nacional ${ }^{31}$, os alunos parecem desconhecer o mesmo. Muitos alunos de medicina espelham-se na figura do professor desde a definição de aspectos éticos e morais, até a influência na escolha da especialidade A correta atitude de docentes em guiar os alunos permitiria ao estudante maior capacidade de resiliência, sendo fundamentais para tornar o ambiente universitário favorável ao aprendizado, prevenindo o sofrimento, sendo extremamente efetivos para diminuir a chance de burnout por parte dos alunos.

Dentre as limitações da pesquisa podemos incluir a não utilização de um questionário padronizado na literatura para avaliação da frustração e pesquisa de dilemas de estudantes universitários, o que limita a comparação com outros trabalhos. Além disso, deve-se considerar a limitação dos estudos quantitativos transversais na avaliação das causas e possíveis consequências que levaram os estudantes à estarem decepcionados e frustrados com o curso de medicina, o que pode ser melhor explorado com estudos qualitativos e de seguimento. Futuras pesquisas que possam enfatizar as consequências e modos de prevenção desses problemas são necessárias, inclusive analisando o novo perfil de aluno e surgimento de novas estratégias de enfrentamento de adversidades geradas pela utilização da metodologia ativa de ensino. 


\section{Conclusão}

Na presente pesquisa os estudantes de medicina mostraram nítida frustração com o curso. Os principais dilemas estiveram relacionados à falta de orientação por parte dos professores e a sobrecarga de atividades acadêmicas.

Nesse sentido, são necessárias mudanças por parte do curso de medicina, tornando mais efetiva a atuação pedagógica em relação aos dilemas dos estudantes. E aplicação de medidas já previstas nas diretrizes curriculares, com o fortalecimento e acessibilidade dos estudantes aos grupos de apoio psicopedagógicos.

\section{Referências}

1. Costa JRB, Romano VF, Costa RR, Gomes AP, Alves LA, Siqueira-Batista R. A transformação curricular e a escolha da especialidade médica. Rev Bras Educ Med. 2014;38(1):47-58. http://dx.doi.org/10.1590/S0100-55022014000100007.

2. Trindade LMDF, Vieira MJ. Medicine students and coping strategies in patient care. Rev Bras Educ Med. 2013;37(2):167-77. http://dx.doi. org/10.1590/S0100-55022013000200003.

3. Al-Dubai SA, Al-Naggar RA, Alshagga MA, Rampal KG. Stress and coping strategies of students in a medical faculty in malaysia. Malays J Med Sci. 2011;18(3):57-64. PMid:22135602.

4. Niemi PM, Vainiomäki PT. Medical students' academic distress, coping, and achievement strategies during the preclinical years. Teach Learn Med. 1999;11(3):125-134. http://dx.doi.org/10.1207/S15328015TL110302.

5. Dyrbye LN, Thomas MR, Shanafelt TD. Medical student distress: causes, consequences, and proposed solutions. Mayo Clin Proc. 2005;80(12):1613-22. http://dx.doi.org/10.4065/80.12.1613. PMid:16342655.

6. Yusoff MS, Abdul Rahim AF, Baba AA, Ismail SB, Mat Pa MN, Esa AR. Prevalence and associated factors of stress, anxiety and depression among prospective medical students. Asian J Psychiatr. 2013;6(2):128-33. http://dx.doi.org/10.1016/j.ajp.2012.09.012. PMid:23466109.

7. Puthran R, Zhang MW, Tam WW, Ho RC. Prevalence of depression amongst medical students: a meta-analysis. Med Educ. 2016;50(4):45668. http://dx.doi.org/10.1111/medu.12962. PMid:26995484.

8. Dyrbye LN, Thomas MR, Shanafelt TD. Systematic review of depression, anxiety, and other indicators of psychological distress among U.S. and canadian medical students. Acad Med. 2006;81(4):354-73. http://dx.doi.org/10.1097/00001888-200604000-00009. PMid:16565188.

9. Tyssen R, Vaglum P, Grønvold NT, Ekeberg $\varnothing$. Suicidal ideation among medical students and young physicians: a nationwide and prospective study of prevalence and predictors. J Affect Disord. 2001;64(1):69-79. http://dx.doi.org/10.1016/S0165-0327(00)00205-6. PMid:11292521.

10. Azad MC, Fraser K, Rumana N, Abdullah AF, Shahana N, Hanly PJ, et al. Sleep disturbances among medical students: a global perspective. J Clin Sleep Med. 2015;11(1):69-74. http://dx.doi.org/10.5664/jcsm.4370. PMid:25515274.

11. Finger G, Silva ER, Falavigna A. Use of methylphenidate among medical students: a systematic review. Rev Assoc Med Bras. 2013;59(3):285-9. http://dx.doi.org/10.1016/j.ramb.2012.10.007. PMid:23680277.

12. Tyssen R, Vaglum P, Grønvold NT, Ekeberg $\varnothing$. Factors in medical school that predict postgraduate mental health problems in need of treatment. A nationwide and longitudinal study. Med Educ. 2001;35(2):110-20. http://dx.doi.org/10.1046/j.1365-2923.2001.00770.x. PMid:11169082.

13. Costa EF, Santana YS, Santos AT, Martins LA, Melo EV, Andrade TM. Sintomas depressivos entre internos de medicina em uma universidade pública brasileira. Rev Assoc Med Bras. 2012;58(1):53-9. http://dx.doi.org/10.1016/S0104-4230(12)70155-0. PMid:22392317.

14. Costa EFO, Rocha MMV, Santos ATRA, Melo EV, Martins LAN, Andrade TM. Common mental disorders and associated factors among final-year healthcare students. Rev Assoc Med Bras. 2014;60(6):525-30. http://dx.doi.org/10.1590/1806-9282.60.06.009. PMid:25650851.

15. Niemi PM, Vainiomäki PT. Medical students' distress--quality, continuity and gender differences during a six-year medical programme. Med Teach. 2006;28(2):136-41. http://dx.doi.org/10.1080/01421590600607088. PMid:16707294.

16. Quintana AM, Rodrigues AT, Arpini DM, Bassi LA, Cecim PS, Santos MS. A angústia na formação do estudante de medicina. Rev Bras Educ Med. 2008;3(2):7-14. http://dx.doi.org/10.1590/S0100-55022008000100002.

17. Oliveira RZ, Gonçalves MB, Bellini LM. Acadêmicos de medicina e suas concepções sobre "ser Médico". Rev Bras Educ Med. 2011;35(3):311-8. http://dx.doi.org/10.1590/S0100-55022011000300003.

18. Benevides-Pereira A, Gonçalves MB. Transtornos emocionais e a formação em Medicina: um estudo longitudinal. Rev Bras Educ Med. 2009;33(1):10-23. http://dx.doi.org/10.1590/S0100-55022009000100003.

19. Binienda J, Schwartz K, Gaspar D. Training in end-of-life care through interaction with dying patients. Acad Med. 2001;76(5):517. http:// dx.doi.org/10.1097/00001888-200105000-00049. PMid:11346556. 
20. Petroianu A, Reis DC, Cunha BD, Souza DM. Prevalência do consumo de álcool, tabaco e entorpecentes por estudantes de medicina da universidade federal de minas gerais. Rev Assoc Med Bras. 2010;56(5):568-71. http://dx.doi.org/10.1590/S0104-42302010000500019. PMid:21152830.

21. Pedrosa AAS, Camacho LAB, Passos SRL, Oliveira RVC. Consumo de álcool entre estudantes universitários. Cad Saude Publica. 2011;27(8):161121. http://dx.doi.org/10.1590/S0102-311X2011000800016. PMid:21877009.

22. Newbury-Birch D, Walshaw D, Kamali F. Drink and drugs: from medical students to doctors. Drug Alcohol Depend. 2001;64(3):265-70. http://dx.doi.org/10.1016/S0376-8716(01)00128-4. PMid:11672941.

23. Grant BF, Dawson DA. Age of onset of drug use and its association with DSM-IV drug abuse and dependence: Results from the national longitudinal alcohol epidemiologic survey. J Subst Abuse. 1998;10(2):163-73. http://dx.doi.org/10.1016/S0899-3289(99)80131-X. PMid:9854701.

24. Baldwin DC Jr, Hughes PH, Conard SE, Storr CL, Sheehan DV. Substance use among senior medical students: a survey of 23 medical schools. JAMA. 1991;265(16):2074-8. http://dx.doi.org/10.1001/jama.1991.03460160052028. PMid:2013926.

25. Tempski P, Bellodi PL, Paro HB, Enns SC, Martins MA, Schraiber LB. What do medical students think about their quality of life? A qualitative study. BMC Med Educ. 2012;12(1):106. http://dx.doi.org/10.1186/1472-6920-12-106. PMid:23126332.

26. Manzar B, Manzar N. To determine the level of satisfaction among medical students of a public sector medical university regarding their academic activities. BMC Res Notes. 2011;4(1):380. http://dx.doi.org/10.1186/1756-0500-4-380. PMid:21974939.

27. Rosenberg DA, Silver HK. Medical student abuse: an unnecessary and preventable cause of stress. JAMA. 1984;251(6):739-42. http://dx.doi. org/10.1001/jama.1984.03340300031023. PMid:6694276.

28. Macedo PNAG, Nardotto LL, Dieckmann LHJ, Ferreira YD, Macedo BAG, Santos MAP, et al. Factors associated with depressive symptoms in a sample of Brazilian medical students. Rev Bras Educ Med. 2009;33(4):595-604. http://dx.doi.org/10.1590/S0100-55022009000400010.

29. O’Neill LD, Wallstedt B, Eika B, Hartvigsen J. Factors associated with dropout in medical education: a literature review. Med Educ. 2011;45(5):440-54. http://dx.doi.org/10.1111/j.1365-2923.2010.03898.x. PMid:21426375.

30. Neumann M, Edelhäuser F, Tauschel D, Fischer MR, Wirtz M, Woopen C, et al. Empathy decline and its reasons: a systematic review of studies with medical students and residents. Acad Med. 2011;86(8):996-1009. http://dx.doi.org/10.1097/ACM.0b013e318221e615. PMid:21670661.

31. Brasil. Senado Federal. Lei n $9394 / 96$ de 20 de dezembro de 1996. Estabelece as diretrizes e bases da educação nacional. Diário Oficial da República Federativa do Brasil, Brasília, DF; 20 dez 1996. p. 27833.

\author{
Autor correspondente \\ Carolina Ribeiro Mainardi \\ Universidade do Estado do Pará - UEPA \\ Centro de Ciências Biológicas e da Saúde, Laboratório de Cirurgia Experimental \\ Tv. Perebebuí, 2623, Bairro do Marco \\ CEP 66087-670, Belém, PA, Brasil \\ Tel.: (91) 3131-1711 \\ E-mail: carolina_mainardi@hotmail.com
}

\title{
Informação sobre os autores
}

DHF é mestrando em cirurgia e pesquisa experimental pela Universidade do Estado do Pará.

CRM e ALV são graduandos do curso de medicina da Universidade do Estado do Pará.

VNY é médico formado pela Universidade do Estado do Pará.

RKCT é mestre em cirurgia e pesquisa experimental pela Universidade do Estado do Pará.

JACS é doutor em bioética e ética em ciências da saúde pela Faculdade de Medicina da Universidade do Porto; professor do Departamento de Saúde Comunitária pela Universidade do Estado do Pará.

\section{Contribuição dos autores}

DHF e CRM foram responsáveis pela concepção, metodologia, coleta de dados, desenvolvimento científico intelectual do estudo, escrita e revisão final; ALV, VNY e RKCT foram responsáveis pela concepção, metodologia, desenvolvimento científico intelectual do estudo, escrita e revisão final; JACS foi responsável pela metodologia, revisão crítica e revisão final.

Todos os autores leram e aprovaram a versão final submetida ao Pará Research Medical Journal. 